\title{
Weak and strong minima : from calculus of variation toward PDE optimization
}

\author{
T. Bayen ${ }^{*}$ and F.J. Silva ${ }^{* *}$ \\ * Université Montpellier 2, CC 051, 34095 Montpellier cedex 5 France; \\ INRA-INRIA 'MODEMIC' team, INRIA Sophia-Antipolis \\ Méditerranée, UMR INRA-SupAgro 729 'MISTEA' 2 place Viala \\ 34060 Montpellier (e-mail: tbayen@math.univ-montp2.fr). \\ ** Laboratoire XLIM-DMI, Université de Limoges, 123 Avenue Albert \\ Thomas 87060 Limoges cedex France (e-mail: \\ francisco.silva@unilim.fr)
}

\begin{abstract}
This note summarizes some recent advances on the theory of optimality conditions for PDE optimization. We focus our attention on the concept of strong minima for optimal control problems governed by semi-linear elliptic and parabolic equations. Whereas in the field of calculus of variations this notion has been deeply investigated, the study of strong solutions for optimal control problems of partial differential equations (PDEs) has been addressed recently. We first revisit some well-known results coming from the calculus of variations that will highlight the subsequent results. We then present a characterization of strong minima satisfying quadratic growth for optimal control problems of semi-linear elliptic and parabolic equations and we end by describing some current investigations.
\end{abstract}

Keywords: Calculus of variation, Optimal Control, Strong minima, Partial differential equations.

\section{INTRODUCTION}

The study of weak and strong minima in calculus of variation goes back to the works of Euler, Legendre, Weierstrass, Jacobi, Erdmann, where the first important results in this area have been stated. The objective of this note is to highlight some new characterizations of strong minima in the field of PDE optimization and their connection with well-known results coming from calculus of variations and optimal control of ordinary differential equations (ODEs).

Let us recall that, in the framework of the calculus of variations, we speak of weak minima whenever neighborhoods of feasible trajectories are considered in the space of Lipschitz functions, whereas for strong minima, neighborhood are considered in the uniform topology. These two definitions lead to different necessary and sufficient conditions. For instance, a necessary first order condition for a weak minima is given by the Euler-Lagrange (EL) equation, which is weaker than Weiestrass-Pontryagin condition, which is necessary for strong minima. Regarding second order sufficient condition, we have that E-L equation and the coercivity of a certain quadratic form $Q_{1}$ imply weak local minima (see Theorem 1), whereas to assure strong local minima essentially the E-L equation has to be replaced by a strict Weiestrass-Pontryagin condition (see Theorem 2).

The concept of weak and strong minima has been considered also in other settings such as the optimal control of ODEs and of semi-linear elliptic and parabolic PDEs. In the ODE case, the study of strong minima, initiated by the seminal work of Pontryagin et al. (1964), is exposed in detail in Milyutin and Osmolovskiu (1998) and the references therein. In this context, important progress has been done in Bonnans and Osmolovskiǔ (2010) and Bonnans and Osmolovskiŭ (2011), in the presence of control and state constraints. For semi-linear elliptic equations, Pontryagin minimum principles have been established for strong minima in Raitums (1986) and Bonnans and Casas $(1989,1991)$. Even if second order theory for weak minima has been deeply studied in Casas et al. (1996); Bonnans (1998); Bonnans and Zidani (1999); Casas and Tröeltzsch (1999, 2002, 2009) and Casas et al. (2008), the first results for the analysis of strong minima are provided in Bayen et al. (2012). In this work a characterization of strong minima satisfying a quadratic growth property is proved. For the case of semi-linear parabolic equations, first-order necessary conditions in the form of a Pontryagin principle has been the subject of study of several works (see the monograph Li and Yong (1994) and the references therein), whereas the second order analysis for weak minima has been investigated for instance in Goldberg and Tröeltzsch (1993); Raymond and Tröltzsch (2000) and Rösch and Tröltzsch (2003).

In this note, we will mainly discuss characterizations of strong minima for optimal control problems governed by semi-linear elliptic and parabolic equations. We present a summary of the work in Bayen et al. (2012) for the elliptic case and we exhibit some recent findings for the parabolic case. A discussion about the current research of the authors is also provided. 
The exposition is organized as follows. In the second section, we make a brief review of the well-known necessary and sufficient optimality conditions in the field of calculus of variations that motivates the analysis for the elliptic and parabolic frameworks. In the third section, we review the results of Bayen et al. (2012) dealing with a characterization of strong minima satisfying quadratic growth in terms of a second order condition and a strengthened Pontryagin condition. Finally, we discuss in the last section extensions of the results in Bayen et al. (2012) to the parabolic case as well as the inclusion of state constraints, which is the subject of our current research.

\section{BOUNDED STRONG MINIMA IN THE CALCULUS OF VARIATIONS}

The well-know results briefly reviewed in this section can be found for instance in e.g. Milyutin and Osmolovskil (1998). We consider the simplest problem in the calculus of variations:

$$
\begin{aligned}
& \inf \int_{a}^{b} \ell(t, y(t), \dot{y}(t)) \mathrm{d} t, \\
& \text { s.t. } y \in W^{1, \infty}\left([a, b] ; \mathbb{R}^{n}\right), \\
& y(a)=\hat{y}_{1}, \quad y(b)=\hat{y}_{2},
\end{aligned}
$$

which can be written as

$$
\left.\begin{array}{l}
\inf J_{1}(u):=\int_{a}^{b} \ell(t, y[u](t), u(t)) \mathrm{d} t, \\
\text { s.t. } \quad u \in L^{\infty}\left([a, b] ; \mathbb{R}^{n}\right), \\
y[u](b)=\hat{y}_{2},
\end{array}\right\}
$$

where

$$
y[u](t):=\hat{y}_{1}+\int_{a}^{t} u(t) \mathrm{d} t \quad \forall t \in(a, b) .
$$

Let us fix $\bar{u} \in L^{\infty}\left([a, b] ; \mathbb{R}^{n}\right)$ and set $\bar{y}:=y[\bar{u}]$.

Definition 2.1. Let $R>0$. We say that $\bar{u}$ is a:

(i) weak local solution of $\left(P_{1}\right)$ if $\exists \varepsilon>0$ such that

$$
J_{1}(\bar{u}) \leq J_{1}(u) \quad \forall u \text { satisfying }\|u-\bar{u}\|_{\infty} \leq \varepsilon .
$$

(ii) strong local solution of $\left(P_{1}\right)$ if $\exists \varepsilon>0$ such that $J_{1}(\bar{u}) \leq J_{1}(u) \quad \forall u$ satisfying $\|y[u]-\bar{y}\|_{\infty} \leq \varepsilon$.

(iii) R-bounded strong local solution of $\left(P_{1}\right)$ if $\exists \varepsilon>0$ such that

$$
\begin{aligned}
& J_{1}(\bar{u}) \leq J_{1}(u) \quad \forall u \text { satisfying } \quad\|u-\bar{u}\|_{\infty} \leq R, \\
& \text { and }\|y[u]-\bar{y}\|_{\infty} \leq \varepsilon .
\end{aligned}
$$

A strong local solution is always a $R$-bounded strong local solution, which itself is always a weak local solution. It is easy to see that a weak local solution is not always a strong one. Take for instance $\ell(t, y, u):=u^{2}-u^{4}$ and $(a, b)=(0,1),\left(\hat{y}_{1}, \hat{y}_{2}\right)=(0,0)$, so that one can see that $\bar{y} \equiv 0$ is a weak minimum but not a $R$-bounded strong one.

Let us define the Hamiltonian $H:[a, b] \times \mathbb{R}^{3 n} \rightarrow \mathbb{R}$, as

$$
H(t, y, p, u):=\ell(t, y, u)+p^{\top} u .
$$

For simplicity we will assume that $H$ is $C^{2}$. Let us recall that if $\bar{u}$ is weak solution of $\left(P_{1}\right)$ then the following E-L condition holds true

where

$$
-\dot{\bar{p}}(t)=H_{y}(t, \bar{y}(t), \bar{p}(t), \bar{u}(t)) \text { a.e. in }(\mathrm{a}, \mathrm{b}),
$$

$$
\bar{p}(t):=-\ell_{u}(t, \bar{y}(t), \bar{u}(t)) \text { a.e. in }(a, b) .
$$

In the above notation and in the rest of this note, the subscripts denote derivation with respect to the corresponding variable. Note that, by definition of $\bar{p}$, we have that

$$
H_{u}(t, \bar{y}(t), \bar{p}(t), \bar{u}(t))=0 \quad \text { a.e. in }(a, b) .
$$

Now, let us define the quadratic form $Q_{1}: L^{2}\left([0, T] ; \mathbb{R}^{n}\right) \rightarrow$ $\mathbb{R}$ by

$$
Q_{1}(v):=\int_{a}^{b} D_{(y, u)^{2}}^{2} H(t, \bar{y}(t), \bar{p}(t), \bar{u}(t))(z[v], v)^{2} \mathrm{~d} t
$$

where

$$
z[v](t):=\int_{a}^{b} v(s) \mathrm{d} s \quad \forall t \in(a, b) .
$$

The following second-order conditions hold true.

Theorem 1. Suppose that $\bar{u}$ is a weak local solution of $\left(P_{1}\right)$. Then $Q_{1}(v) \geq 0$ for all $v$ such that $z[v](b)=0$. Conversely, if there exists $\alpha>0$ such that $Q_{1}(v) \geq \alpha\|v\|_{2}^{2}$ for all $v$ such that $z[v](b)=0$, then $\bar{u}$ is a weak solution of $\left(P_{1}\right)$.

Now, we recall the analogous analysis for $R$-bounded strong local solutions. If $\bar{u}$ is a $R$-bounded strong local solution of $\left(P_{1}\right)$ then it satisfies a.e. the following Weierstrass-Pontryagin condition

$$
H(t, \bar{y}(t), \bar{p}(t), \bar{u}(t)) \leq H(t, \bar{y}(t), \bar{p}(t), v) \quad \forall v \in \mathbb{R}^{n} .
$$

We say that the strict Weierstrass-Pontryagin condition holds at $\bar{u}$ if the above inequality can be replaced by a strict inequality. Note that (4) is a stronger condition than (3). We have the following second-order sufficient condition for a $R$-bounded strong local solution of $\left(P_{1}\right)$.

Theorem 2. Suppose that $\bar{u}$ is continuous and that

(i) The strict Weierstrass-Pontryagin condition holds true.

(ii) If $z[v](b)=0$ then $Q(v) \geq \alpha\|v\|_{2}^{2}$.

Then, $\bar{u}$ is a $R$-bounded strong local solution of $\left(P_{1}\right)$.

Remark 3. An extension of this result when $\bar{u}$ is not continuous can be found in Milyutin and Osmolovskil (1998).

\section{SEMI-LINEAR ELLIPTIC CONTROLLED SYSTEMS}

In this part, we summarize a result of Bayen et al. (2012) concerning the characterization of strong minima for optimal control problems governed by semi-linear elliptic equations. Although it is more delicate to study strong minima in this setting, the scheme of the analysis is parallel to the one in the calculus of variations theory.

Let $\Omega \subset \mathbb{R}^{n}$ a bounded open set with a $C^{1,1}$ boundary. We consider the semi-linear elliptic equation:

$$
\left\{\begin{aligned}
-\Delta y+\varphi(y, u) & =0 & & \text { in } \Omega, \\
y & =0 & & \text { on } \partial \Omega .
\end{aligned}\right.
$$

Whenever $\varphi$ is Lipschitz and satisfies $\varphi_{y} \geq 0$, it is well known that for any $u \in L^{\infty}(\Omega)$, there exists a unique solution $y[u] \in W^{2, s}(\Omega) \cap W_{0}^{1, s}(\Omega)$ (with $\left.s \in(1, \infty)\right)$ ) of (5). In particular, as $s$ is arbitrary, classical Sobolev inequalities yield that $y[u] \in C(\bar{\Omega})$. Let us now take $a, b \in C(\bar{\Omega})$ such that $a \leq b$ over $\bar{\Omega}$. Consider the set

$$
\mathcal{K}_{1}=\left\{u \in L^{\infty}(\Omega) \mid a(x) \leq u(x) \leq b(x), \text { a.e. }\right\},
$$

and a cost function $\ell(y, u)$ satisfying standard assumptions (see Bayen et al. (2012)). We define 


$$
J_{2}(u):=\int_{\Omega} \ell(y[u](x), u(x)) \mathrm{d} x,
$$

and our aim is to study the problem:

$$
\min J_{2}(u) \quad \text { s.t. } u \in \mathcal{K}_{1} \text {. }
$$

Definition 3.1. Let $\bar{u} \in \mathcal{K}_{1}$ and set $\bar{y}:=y[\bar{u}]$. We say that (i) $\bar{u}$ is a weak local solution of $\left(P_{2}\right)$ if $\exists \varepsilon>0$ such that

$$
J_{2}(\bar{u}) \leq J_{2}(u) \forall u \in \mathcal{K}_{1} \text { s.t. }\|u-\bar{u}\|_{\infty} \leq \varepsilon .
$$

(ii) $\bar{u}$ is a strong local solution of $\left(P_{2}\right)$ if $\exists \varepsilon>0$ such that

$$
J_{2}(\bar{u}) \leq J_{2}(u) \forall u \in \mathcal{K}_{1} \text { s.t. }\|y[u]-\bar{y}\|_{\infty} \leq \varepsilon .
$$

Next we define stronger notions involving quadratic growths.

Definition 3.2. We say that:

(i) $J_{2}$ has a local quadratic growth in $\mathcal{K}_{1}$ at $\bar{u}$ in the weak sense if $\exists \alpha, \varepsilon>0$ s.t.

$J_{2}(\bar{u})+\alpha\|u-\bar{u}\|_{2}^{2} \leq J_{2}(u) \forall u \in \mathcal{K}_{1}$ s.t. $\|u-\bar{u}\|_{\infty} \leq \varepsilon$.

(ii) $J_{2}$ has a local quadratic growth in $\mathcal{K}_{1}$ at $\bar{u}$ in the strong sense if $\exists \alpha, \varepsilon>0$ s.t.

$J_{2}(\bar{u})+\alpha\|u-\bar{u}\|_{2}^{2} \leq J_{2}(u) \quad \forall u \in \mathcal{K}_{1}$ s.t. $\|y[u]-\bar{y}\|_{\infty} \leq \varepsilon$.

We introduce the Hamiltonian

$$
H(y, p, u):=\ell(y, u)-p \varphi(y, u) .
$$

The adjoint state $\bar{p}$ is defined as the unique solution of

$$
\left\{\begin{aligned}
-\Delta \bar{p} & =H_{y}(\bar{y}, \bar{p}, \bar{u}) & & \text { in } \Omega, \\
\bar{p} & =0 & & \text { on } \partial \Omega .
\end{aligned}\right.
$$

Definition 3.3. We say that

(i) $\bar{u}$ is a Pontryagin extremal if a.e.

$H(\bar{y}(x), \bar{p}(x), \bar{u}(x)) \leq H(\bar{y}(x), \bar{p}(x), v), \quad \forall v \in[a(x), b(x)]$

(ii) $H$ has global quadratic growth at $\bar{u} \in \mathcal{K}_{1}$ if $\exists \alpha>0$ s.t.

$H(\bar{y}(x), \bar{p}(x), \bar{u}(x))+\alpha|v-\bar{u}(x)|^{2} \leq H(\bar{y}(x), \bar{p}(x), v)$,

for all $x \in \bar{\Omega}$ and $v \in[a(x), b(x)]$.

First and second-order necessary and sufficient conditions for a weak local solution $\bar{u}$ can be found e.g. in Bonnans (1998) and Casas et al. (1996).

Regarding strong local solutions, a first order necessary condition of Pontryagin type has been proved in Raitums (1986) and Bonnans and Casas (1991, 1989). Namely, if $\bar{u}$ is a strong local solution of $\left(P_{2}\right)$, then it is a Pontryagin extremal.

Our main result is a characterization of the local quadratic growth $J$ in the strong sense, which is based on a secondorder condition. Let us define the tangent cone to $\mathcal{K}_{1}$ at $\bar{u}$ by

$$
T_{\mathcal{K}_{1}}(\bar{u}):=\left\{\begin{array}{ll}
v \in L^{2}(\Omega) ; & v \geq 0 \text { in }[\bar{u}(x)=a(x)] \\
& v \leq 0 \text { in }[\bar{u}(x)=b(x)]
\end{array}\right\},
$$

and the critical cone at $\bar{u}$ :

$$
C_{\mathcal{K}_{1}}(\bar{u}):=\left\{v \in T_{\mathcal{K}_{1}}(\bar{u}) ; H_{u}(\bar{y}(x), \bar{p}(x), \bar{u}(x)) v(x)=0,\right.
$$

Let us consider the quadratic form

$$
Q_{2}(v):=\int_{\Omega} D_{(y, u)^{2}}^{2} H(\bar{y}(x), \bar{p}(x), \bar{u}(x))(z[v], v)^{2} \mathrm{~d} x,
$$

where $z[v]$ is the solution of

$$
\left\{\begin{aligned}
-\Delta z[v]+D \varphi(\bar{y}, \bar{u})(z[v], v) & =0 & & \text { in } \Omega, \\
z[v] & =0 & & \text { on } \partial \Omega .
\end{aligned}\right.
$$

Now we can state the main result of Bayen et al. (2012).
Theorem 4. The cost functional $J$ satisfies the local quadratic growth property at $\bar{u}$ in the strong sense if and only if the Hamiltonian has global quadratic growth at $\bar{u} \in \mathcal{K}_{1}$ and there exists $\alpha>0$ s.t.

$$
Q_{2}(v) \geq \alpha\|v\|_{2}^{2} \quad \text { for all } v \in C_{\mathcal{K}_{1}}(\bar{u})
$$

The proof of the above theorem relies on a decomposition result of the cost functional which extends the one in Bonnans and Osmolovskil (2010) for the ODE framework. It provides a second-order expansion for $J_{2}$, which can be written as the sum of two terms: the first one corresponds to a variation of the cost due to a perturbation which is "large in $L^{\infty}$ ", but supported on a set with "small" measure. The second term corresponds to a variation which is "small in $L^{\infty}$ " and thus can be treated as in the classical weak case. The proof of this decomposition result relies on standard estimates in $L^{s}$ for solutions of linear elliptic equations (see e.g. Gilbarg and Trudinger (1983)).

\section{SEMI-LINEAR PARABOLIC CONTROLLED SYSTEMS}

Let $\Omega \subseteq \mathbb{R}^{n}$ open and bounded with a $C^{1,1}$ boundary, and let $T>0$ be fixed. Set $Q=\Omega \times(0, T)$ and $\Sigma=\partial \Omega \times(0, T)$. Let us consider the following controlled parabolic equation

$$
\begin{aligned}
\partial_{t} y-\Delta y+\varphi(y, u) & =0, \quad \text { in } Q, \\
y(0, \cdot) & =y_{0}(\cdot), \quad \text { in } \Omega, \\
y(t, x) & =0, \quad \text { in } \Sigma,
\end{aligned}
$$

where $\varphi$ is Lipschitz and satisfies $\varphi_{y} \geq 0$. Classical regularity assumptions for $\varphi$ imply that for any $u \in$ $L^{\infty}(Q)$ equation $(10)$ admits a unique solution $y[u] \in$ $L^{2}\left([0, T] ; H^{1}(\Omega)\right) \cap C(\bar{Q})$.

Given $a, b \in C(\bar{Q})$, let us define

$$
\mathcal{K}_{2}=\left\{u \in L^{\infty}(Q) \mid a(t, x) \leq u(t, x) \leq b(t, x) \text {, a.e. }\right\} \text {. }
$$
and

$$
\mathcal{K}_{3}=\left\{\begin{aligned}
u \in L^{\infty}(Q) ; & G_{i}(u)=0 i=1, \ldots, k, \\
G_{j}(u) & \leq 0 j=k+1, \ldots, m
\end{aligned}\right\} .
$$

where, for some $C^{2}$ functions $g_{i}, g_{j}$,

$G_{i}(u):=\int_{\Omega} g_{i}(y[u](T, x)) \mathrm{d} x, \quad G_{j}(u):=\int_{\Omega} g_{j}(y[u](T, x)) \mathrm{d} x$.

Given $C^{2}$ functions $\ell: \mathbb{R}^{2} \rightarrow \mathbb{R}$ and $\Phi: \mathbb{R} \rightarrow \mathbb{R}$ we set

$J_{3}(u):=\int_{Q} \ell(y[u](t, x), u(t, x)) \mathrm{d} x \mathrm{~d} t+\int_{\Omega} \Phi(y[u](T, x)) \mathrm{d} x$.

Using sharp results from the theory of anisotropic Sobolev spaces (see Besov et al. (1979)), we can show a decomposition result for the cost function in the same line of Bonnans and Osmolovskiu (2010) and Bayen et al. (2012). This allows to prove the natural extension of Theorem 4 for the problem

$$
\min J_{3}(u) \quad \text { s.t. } u \in \mathcal{K}_{2} .
$$

We are now studying the validity of this type of characterization for the more complicated problem when final state constraints are included, i.e.

$$
\min J_{3}(u) \quad \text { s.t. } u \in \mathcal{K}_{2} \cap \mathcal{K}_{3} .
$$

The main difficulty is to prove in this case that the coercivity of the associated quadratic form on the critical cone is a necessary condition for quadratic growth in the strong sense. Some preliminary advances in this direction 
have been done following ideas from Bonnans and Zidani (1999) in the elliptic framework.

\section{ACKNOWLEDGEMENTS}

The first author thanks INRIA for providing him a one year research opportunity at INRA-INRIA project MODEMIC.

\section{REFERENCES}

Bayen, T., Bonnans, J., and Silva, F.J. (2012). Characterization of local quadratic growth for strong minima in the optimal control of semi-linear elliptic equations. accepted in to Trans. Amer. Math. Society.

Besov, O., Il'in, V.P., and Nikol'skil, S.M. (1979). Integral Representations of Functions and Imbedding Theorems. John Wiley and Sons Inc.

Bonnans, J.F. (1998). Second order analysis for control constrained optimal control problems of semilinear elliptic systems. Appl. Math. Optim., 38-3, 303-325.

Bonnans, J. and Casas, E. (1989). Maximum principles in the optimal control of semilinear elliptic systems. 5th IFAC Conference on Distributed Parameter Systems, A. El Jai ed. (Perpignan), Pergamon Press, 33-1, 274-298.

Bonnans, J. and Casas, E. (1991). Un principe de Pontryagin pour le contrôle des systèmes semilinéaires elliptiques. Journal of Differential Equations, 90, 288-303.

Bonnans, J. and Osmolovskiu,, N. (2010). Second-order analysis of optimal control problems with control and initial-final state constraints. Journal of Convex Analysis, 17(3), 885-913.

Bonnans, J. and Osmolovskiŭ, N. (2011). Characterization of a local quadratic growth of the hamiltonian for control constrained optimal control problems. Dynamics of Continuous, Discrete and Impulsive Systems. To appear.

Bonnans, J. and Zidani, H. (1999). Optimal control problems with partially polyhedric constraints. SIAM J. Control Optim., 37(6), 1726-1741 (electronic).

Casas, E., de Los Reyes, J., and Tröeltzsch, F. (2008). Sufficient second-order optimality conditions for semilinear control problems with pointwise state constraints. SIAM J. Optim., 19-2, 616-643.

Casas, E. and Tröeltzsch, F. (1999). Second order necessary optimality conditions for some state-constrained control problems of semilinear elliptic equations. Appl. Math. Optim., 39, 211-228.

Casas, E. and Tröeltzsch, F. (2002). Second order necessary and sufficient optimality conditions for optimization problems and applications to control theory. SIAM J. Optim., 13, 406-431.

Casas, E. and Tröeltzsch, F. (2009). First- and secondorder optimality conditions for a class of optimal control problems with quasilinear elliptic equations. SIAM J. Control Optim., 48-2, 688-718.

Casas, E., Tröeltzsch, F., and Unger, A. (1996). Second order sufficient optimality conditions for a nonlinear elliptic control problem. Journal for Analysis and its Applications, 15, 687-707.

Gilbarg, D. and Trudinger, N. (1983). Elliptic partial differential equations of second order, 2nd edition. Springer Verlag, Berlin.
Goldberg, H. and Tröeltzsch, F. (1993). Second order sufficient optimality conditions for a class of non-linear parabolic boundary control problems. SIAM J. Control Optim., 31, 1007-1025.

Li, X. and Yong, J. (1994). Optimal Control Theory for Infinite Dimensional Systems. Birkhuser. Systems and Control: Foundations and Applications.

Milyutin, A. and Osmolovskiı̌, N. (1998). Calculus of Variations and Optimal Control. AMS. Systems and Control: Foundations and Applications.

Pontryagin, L., Boltyanskiy, V., Gamkrelidze, R., and Mishchenko, E. (1964). Mathematical theory of optimal processes. The Macmillan Company.

Raitums, U.E. (1986). The maximum principle in optimal control problems for an elliptic equation. Z. Anal. Anwendungen, 5(4), 291-306.

Raymond, J. and Tröltzsch, F. (2000). Second order sufficient optimality conditions for nonlinear parabolic control problems with state constraints. Discrete and Continuous Dynamical Systems, 6, 431-450.

Rösch, A. and Tröltzsch, F. (2003). Sufficient second order optimality conditions for a parabolic optimal control problem with pointwise control-state constraints. SIAM J. Control Optim., 42, 138-154. 